\title{
Classic congenital adrenal hyperplasia due to 21-hydroxylase deficiency, salt wasting form
}

INSERM

\section{Source}

INSERM. (1999). Orphanet: an online rare disease and orphan drug data base. Classic congenital adrenal hyperplasia due to 21-hydroxylase deficiency, salt wasting form. ORPHA:315306

The salt wasting form of classic congenital adrenal hyperplasia due to 21-hydroxylase deficiency (classical 21 OHD CAH; see this term) is characterized by virilization of the external genitalia in females, hypocortisolism, precocious pseudopuberty and renal salt loss due to aldosterone deficiency. 